\title{
ON THE PRESERVATION OF DETERMINACY UNDER CONVOLUTION
}

\author{
CHRISTIAN BERG
}

\begin{abstract}
In 1959 Devinatz remarked that if $\mu * \nu$ is a determinate measure on the real line, then so are $\mu$ and $\nu$. It is shown here how this follows from a theorem of $\mathrm{M}$. Riesz, and also how it can be extended to $d$ dimensions. Recently Diaconis raised the question whether the converse is true. We answer this in the negative by producing a determinate measure $\nu$ on the real line such that $\nu * \nu$ is indeterminate. The relation to previous work of Heyde and to the condition of Carleman is discussed.
\end{abstract}

1. The positive result. Let $\mathscr{M}^{*}\left(\mathbf{R}^{d}\right)$ denote the set of positive Borel measures on $\mathbf{R}^{d}$ having moments of all orders. For $\mu \in \mathscr{M}^{*}\left(\mathbf{R}^{d}\right)$ the moment sequence $s_{n}(\mu)$ is defined by

$$
s_{n}(\mu)=\int_{\mathbf{R}^{d}} x^{n} d \mu(x) \text { for } n=\left(n_{1}, \ldots, n_{d}\right) \in \mathbf{N}_{0}^{d},
$$

where $x^{n}=x_{1}^{n_{1}} \cdots x_{d}^{n_{d}}$. For $\mu, \nu \in \mathscr{M}^{*}\left(\mathbf{R}^{d}\right)$ we write $\mu \sim \nu$ if $\mu$ and $\nu$ have the same moments, i.e. if $s_{n}(\mu)=s_{n}(\nu)$ for all $n \in \mathbf{N}_{0}^{d}$. The equivalence class containing $\mu$ is denoted $[\mu]$, and we recall that $\mu$ is called determinate if $[\mu]=\{\mu\}$, and indeterminate otherwise. In the indeterminate case $[\mu]$ is a convex set, and it is not difficult to see that $[\mu]$ is compact in the weak topology. We shall also need the following result which is a special case of a theorem of Douglas [7], and for $d=1$ it is usually attributed to Naimark (cf. Akhiezer [1, p. 47]).

1.1. Proposition. Let $\mu \in \mathscr{M}^{*}\left(\mathbf{R}^{d}\right)$. Then $\mu$ is an extreme point of $[\mu]$ if and only if the polynomials are dense in $L^{1}\left(\mathbf{R}^{d}, \mu\right)$. In particular, the polynomials are dense in $L^{1}\left(\mathbf{R}^{d}, \mu\right)$ in case $\mu$ is determinate.

For $\mu \in \mathscr{M}^{*}\left(\mathbf{R}^{d}\right)$ the Fourier transform $\hat{\mu}: \mathbf{R}^{d} \rightarrow \mathbf{C}$ is a $C^{\infty}$-function given by

$$
\hat{\mu}(y)=\int_{\mathbf{R}^{d}} e^{i\langle x, y\rangle} d \mu(x), \quad y \in \mathbf{R}^{d} .
$$

The zero set $\{\hat{\mu}=0\}$ may of course have interior points since it is even possible that $\hat{\mu}$ has compact support. We have, however, the following result:

1.2. Proposition. Assume that $\mu \in \mathscr{M}^{*}\left(\mathbf{R}^{d}\right) \backslash\{0\}$ is an extreme point of $[\mu]$. Then $\{\hat{\mu}=0\}$ has no interior points, and for $d=1$ then $\{\hat{\mu}=0\}$ is a discrete set.

Received by the editors September 12, 1983 and, in revised form, March 5, 1984.

1980 Mathematics Subject Classification. Primary 60E05, 44A60; Secondary 26E10, 60E07.

Key words and phrases. Determinate probability, Nevanlinna extremal measure, infinitely divisible measure, Carleman's condition. 
Proof. Assume that $\hat{\mu}$ is identically zero in an open neighbourhood of $y_{0} \in \mathbf{R}^{d}$. Then also

$$
D^{\alpha} \hat{\mu}(y)=i^{|\alpha|} \int_{\mathbf{R}^{d}} x^{\alpha} e^{i\langle x, y\rangle} d \mu(x)=0
$$

for all $y$ in this neighbourhood and all $\alpha \in \mathbf{N}_{0}^{d}$, and this implies in particular that

$$
\int_{\mathbf{R}^{d}} p(x) e^{i\left\langle x, y_{0}\right\rangle} d \mu(x)=0
$$

for all polynomials $p$. Since $e^{i\left\langle x, y_{0}\right\rangle}$ is bounded and $\mu$ is an extreme point of $[\mu]$, we get by Proposition 1.1 that $e^{i\left\langle x, y_{0}\right\rangle}$ is zero $\mu$-almost everywhere, which is a contradiction.

In the case $d=1$ we also prove by contradiction that $\{\hat{\mu}=0\}$ is discrete. In fact, if $\{\hat{\mu}=0\}$ has an accumulation point $y_{0}$ we can find a sequence $\left(y_{n}\right)$ of mutually different points from $\{\hat{\mu}=0\}$ converging to $y_{0}$, and we may assume that it is either increasing or decreasing. By the mean-value theorem this sequence is separated by a sequence $\left(\xi_{n}\right)$ converging to $y_{0}$ such that $(\operatorname{Re} \hat{\mu})^{\prime}\left(\xi_{n}\right)=0$, hence $(\operatorname{Re} \hat{\mu})^{\prime}\left(y_{0}\right)=0$. Repeated applications of the mean-value theorem show that $(\operatorname{Re} \hat{\mu})^{(n)}\left(y_{0}\right)=0$ for all $n$ and, similarly, $(\operatorname{Im} \hat{\mu})^{(n)}\left(y_{0}\right)=0$. Therefore

$$
\int(i x)^{n} e^{i x y_{0}} d \mu(x)=0 \text { for all } n \geqslant 0,
$$

and as above this leads to a contradiction.

If $\mu, \nu \in \mathscr{M}^{*}\left(\mathbf{R}^{d}\right)$ then the binomial formula implies that $\mu * \nu \in \mathscr{M}^{*}\left(\mathbf{R}^{d}\right)$ and

$$
s_{n}(\mu * \nu)=\sum_{0 \leqslant p \leqslant n}\left(\begin{array}{l}
n \\
p
\end{array}\right) s_{p}(\mu) s_{n-p}(\nu), \quad n \in \mathbf{N}_{0}^{d},
$$

with the usual notation

$$
\left(\begin{array}{l}
n \\
p
\end{array}\right)=\left(\begin{array}{l}
n_{1} \\
p_{1}
\end{array}\right) \cdots\left(\begin{array}{l}
n_{d} \\
p_{d}
\end{array}\right) \text { for } 0 \leqslant p_{i} \leqslant n_{i}, \quad i=1, \ldots, d .
$$

The following result is, for $d=1$, due to Devinatz [5].

1.3. THEOREM. Let $\mu, \nu \in \mathscr{M}^{*}\left(\mathbf{R}^{d}\right)$ be such that $\mu * \nu$ is determinate. Then $\mu$ and $\nu$ are determinate.

Proof. Assume that $\mu$ is indeterminate. By the Krein-Milman theorem there are at least two different extreme points $\mu_{1}, \mu_{2}$ in [ $\mu$ ]. If $\nu$ is indeterminate we choose an extreme point $\nu_{1} \in[\nu]$, and if $\nu$ is determinate we put $\nu_{1}=\nu$. Then $\mu_{1} * \nu_{1} \sim \mu_{2} * \nu_{1}$ $\sim \mu * \nu$, so $\mu_{1} * \nu_{1}=\mu_{2} * \nu_{1}$ because $\mu * \nu$ is assumed to be determinate. Then $\left(\hat{\mu}_{1}-\hat{\mu}_{2}\right) \hat{\nu}_{1} \equiv 0$, but by Proposition 1.2, $\left\{\hat{\nu}_{1} \neq 0\right\}$ is an open dense subset of $\mathbf{R}^{d}$, and we get $\hat{\mu}_{1} \equiv \hat{\mu}_{2}$, hence $\mu_{1}=\mu_{2}$, which is a contradiction.

1.4. Remark. The essential part of the proof of Proposition 1.2 is the essential part of the justification that A. Devinatz had in 1959 for the validity of his remark concerning $\mu * \nu$. He has kindly conveyed this to me with permission to present it. In an earlier version of the present paper Theorem 1.3 was proved for $d=1$ by means of the following result of independent interest:

1.5. Proposition. Let $\mu \in \mathscr{M}^{*}(\mathbf{R})$ be indeterminate. For every $a \in \mathbf{R} \backslash\{0\}, C_{a}=$ $\{\hat{\boldsymbol{\sigma}}(a) \mid \boldsymbol{\sigma} \in[\mu]\}$ is a compact convex set in $\mathbf{C}$ with nonempty interior. 
Proof. Since $[\mu]$ is a compact convex set in the weak topology, it is clear that $C_{a}$ is compact and convex. Let $P$ denote the vector space of real polynomials and consider the positive linear functional $L: P \rightarrow \mathbf{R}$ defined by $L(p)=\int p d \mu$. If $f: \mathbf{R} \rightarrow \mathbf{R}$ is continuous and bounded in absolute value by a polynomial, $M$. Riesz [13] defined

$$
\begin{aligned}
& \underline{L}(f)=\sup \{L(p) \mid p \in P, p \leqslant f\}, \\
& \bar{L}(f)=\inf \{L(p) \mid p \in P, f \leqslant p\} .
\end{aligned}
$$

Clearly $-\infty<\underline{L}(f) \leqslant \bar{L}(f)<\infty$ and $\underline{L}(f)=\bar{L}(f)$ if $f$ is a polynomial. By the Hahn-Banach theorem it is easy to see that $\underline{L}(f)<\bar{L}(f)$ for at least one $f \notin P$, since $\mu$ is indeterminate and

$$
[\underline{L}(f), \bar{L}(f)]=\left\{\int f d \sigma \mid \sigma \sim \mu\right\} .
$$

A deeper result of $\mathrm{M}$. Riesz [13, §27] asserts that $\underline{L}(f)<\bar{L}(f)$ for all continuous functions $f \notin P$ which are bounded in absolute value by a polynomial. In particular, $\left\{\int \cos (a x-\theta) d \sigma(x) \mid \sigma \in[\mu]\right\}$ is an interval of positive length for each $\boldsymbol{\theta} \in \mathbf{R}$ and this length is the width of $C_{a}$ in the direction $e^{i \theta}$. This shows that $C_{a}$ has interior points.

It is clear how Theorem 1.3 in case $d=1$ may be deduced from Proposition 1.5. In fact, if $\mu \in \mathscr{M}^{*}(\mathbf{R})$ is indeterminate and $\nu \in \mathscr{M}^{*}(\mathbf{R}) \backslash\{0\}$, we see that $\mu * \nu$ is indeterminate by first choosing $a \in \mathbf{R} \backslash\{0\}$ such that $\hat{\nu}(a) \neq 0$, and we then choose $\sigma \sim \mu$ such that $\hat{\mu}(a) \neq \hat{\sigma}(a)$ according to Proposition 1.5. Then $\mu * \nu$ and $\sigma * \nu$ are equivalent but different.

1.6. Remarks. (a) It is easy to show that if $\mu \in \mathscr{M}^{*}(\mathbf{R})$ is indeterminate then $\mu * \mu$ is indeterminate. Indeed, if $\mu$ is indeterminate then for any real $t$ there is a measure $\sigma_{t} \sim \mu$ with an atom at $t$ (see [15, p. 60 or Theorem B in 10]). Thus $\sigma_{t} * \sigma_{t}$ has an atom in $2 t$ and is equivalent to $\mu * \mu$, which then has to be indeterminate.

(b) As an application of Proposition 1.5 we see that if $\mu \in \mathscr{M}^{*}(\mathbf{R})$ is indeterminate and symmetric (hence $s_{n}(\mu)=0$ for $n$ odd), there exist nonsymmetric measures with the same moments. In fact, if all the measures in $[\mu]$ were symmetric then $C_{a} \subseteq \mathbf{R}$ for all $a \in \mathbf{R}$. This fact has been noticed by Heyde [9].

2. The negative result. The following answers in the negative a question raised by Diaconis and Ylvisaker [6] in a problem arising in statistics. See also [8].

2.1. THEOREM. There exists a determinate measure $\nu \in \mathscr{M}^{*}(\mathbf{R})$ such that $\nu * \nu$ is indeterminate.

We shall make use of the Nevanlinna extremal measures (short: $\mathrm{N}$-extremal). If $\mu \in \mathscr{M}^{*}(\mathbf{R})$ is indeterminate, Nevanlinna gave in 1922 a parametrization of the compact convex set $[\mu]$ by means of the holomorphic functions mapping the upper half-plane into itself. The constant functions $z \mapsto t, t \in \mathbf{R} \cup\{\infty\}$, parametrize the $\mathrm{N}$-extremal measures in $[\mu]$. A famous theorem of $\mathbf{M}$. Riesz characterizes the $\mathrm{N}$-extremal measures as the measures $\sigma \in[\mu]$ for which the set of polynomials $P$ is dense in $L^{2}(\mathbf{R}, \sigma)$. By comparison with Proposition 1.1 we see that the $\mathrm{N}$-extremal measures form a (compact) subset of the extreme points of $[\mu]$. It is known that 
$\mathrm{N}$-extremal measures are discrete with countably many atoms. For these results see Shohat-Tamarkin [15, pp. 57-65] or Akhiezer [1, p. 98]. Recent treatments may be found in Landau [10] and Buchwalter and Cassier [4].

We also need the notion of $m$-canonical measures in $[\mu]$. They are parametrized by the holomorphic functions which are rational of degree $m$, so that 0 -canonical measures and $\mathrm{N}$-extremal measures are the same (see Akhiezer [1, p. 115]). Recently Buchwalter and Cassier [3] have characterized the $m$-canonical measures $\sigma \in[\mu]$ as those for which the closure of the polynomials in $L^{2}(\sigma)$ is of codimension $m$.

Proof of Theorem 2.1. Let $\mu \in \mathscr{M}^{*}(\mathbf{R})$ be an N-extremal indeterminate measure. By the theorem of perturbation [2, Theorem 8], a change of position and size of finitely many atoms of $\mu$ will provide a new $\mathrm{N}$-extremal measure. We may therefore assume that $\mu$ has the form $\mu=\varepsilon_{0}+\varepsilon_{1}+\varepsilon_{2}+\sigma$, where $\sigma$ is a discrete measure with support not containing $0,1,2$. The measure $\nu=\varepsilon_{0}+\varepsilon_{1}+\sigma$ is determinate (cf. [2, Theorem 7]) and $\nu * \nu=\mu+\tau$ for some positive measure $\tau$. If $\mu_{1} \sim \mu$ then $\mu_{1}+\tau \sim$ $\mu+\tau$, which shows that $\nu * \nu$ is indeterminate.

In the following we need an example of a measure with similar properties as above, stated in the following lemma.

2.2. Lemma. There exists a determinate probability $\sigma \in \mathscr{M}^{*}(\mathbf{R})$ such that $x^{2} \sigma$ is determinate and $\sigma * \sigma * \sigma$ is indeterminate.

Proof. If $\mu$ is $\mathrm{N}$-extremal with no atom at zero then $x^{2} \mu$ is 1 -canonical. In fact, if $k_{0} \in L^{2}(\mu)$ is such that $\int p(x) k_{0}(x) d \mu(x)=p(0)$ for all $p \in P$ (see [2, formula (3)]), then $f(x)=k_{0}(x) / x \in L^{2}\left(x^{2} \mu\right)$ and

$$
\int p(x) f(x) x^{2} d \mu(x)=\int x p(x) k_{0}(x) d \mu(x)=0
$$

showing that $\operatorname{dim}\left(L^{2}\left(x^{2} \mu\right) / \bar{P}\right) \geqslant 1$. Conversely, if $\varphi \in L^{2}\left(x^{2} \mu\right)$ is orthogonal on $P$, then, defining $\lambda=\int x \varphi(x) d \mu(x)$, we have for $p \in P$,

$$
\begin{aligned}
\int p(x)\left(x \varphi(x)-\lambda k_{0}(x)\right) d \mu(x) & =\int(p(x)-p(0)) x \varphi(x) d \mu(x) \\
& =\int q(x) \varphi(x) x^{2} d \mu(x)=0
\end{aligned}
$$

because $q(x):=x^{-1}(p(x)-p(0)) \in P$. This shows that $x \varphi(x)=\lambda k_{0}(x) \mu$-a.e. so that finally $\operatorname{dim}\left(L^{2}\left(x^{2} \mu\right) / \bar{P}\right)=1$.

Let $\mu=\varepsilon_{0}+\varepsilon_{1}+\varepsilon_{2}+\varepsilon_{3}+\sigma$ be an $\mathrm{N}$-extremal measure where the support of $\sigma$ is different from $\{0,1,2,3\}$. Then $\nu=\varepsilon_{0}+\varepsilon_{1}+\sigma$ is determinate and $\nu^{* 3}$ is indeterminate since it majorizes $\mu$. For any $a \in \mathbf{R}$ we have that $\nu * \varepsilon_{a}$ is determinate and $\left(\nu * \varepsilon_{a}\right)^{* 3}$ is indeterminate, and we choose $a$ so small that $0 \notin \operatorname{supp}\left(\mu * \varepsilon_{a}\right)$. Then $x^{2}\left(\mu * \varepsilon_{a}\right)$ is 1-canonical, so by the theorem of subtraction by Buchwalter and Cassier (see [3, Théorème 4]), $x^{2}\left(\nu * \varepsilon_{a}\right)$ is determinate. The measure $\sigma=$ $\nu(\mathbf{R})^{-1}\left(\nu * \varepsilon_{a}\right)$ has the desired properties.

2.3. Applications. (a) The proof of Theorem 2.1 also shows that the $\mathrm{N}$-extremal measure $\mu$ is the sum of two determinate measures $\varepsilon_{0}$ and $\nu$. This result was used in 
Petersen [12] to construct a determinate measure in $\mathbf{R}^{2}$ with indeterminate marginal distributions.

(b) There are probabilities $\mu$ and $\sigma$, say on $[0, \infty[$, with $\mu$ indeterminate, $\sigma$ determinate and yet $s_{n}(\mu) \leqslant s_{n}(\sigma)$ for all $n \geqslant 0$. In fact if $\mu$ is an $N$-extremal probability on [0, $\infty$ [ of the form $\mu=\frac{1}{2} \varepsilon_{0}+\frac{1}{2} \sigma$ and 0 is not an atom of $\sigma$, then $s_{n}(\mu)=\frac{1}{2} s_{n}(\sigma)$ for $n \geqslant 1$.

(c) The construction of Lemma 2.2 can be used to produce a counterexample to a statement of Heyde [9, Corollary 2], which in corrected form says: There exists an infinitely divisible and indeterminate probability $\mu$ such that $\mu$ is the only infinitely divisible measure in $[\mu]$.

To see this we recall Kolmogorov's canonical representation of an infinitely divisible probability $\mu \in \mathscr{M}^{*}(\mathbf{R})$ :

$$
\log \hat{\mu}(y)=i c y+\int_{-\infty}^{\infty}\left(e^{i y x}-1-i y x\right) x^{-2} d \lambda(x),
$$

where $c \in \mathbf{R}$ and $\lambda \in \mathscr{M}^{*}(\mathbf{R})$ are uniquely determined by $\mu$ (cf., e.g., Lukacs [11, p. 90]). Note that

$$
\frac{1}{i^{n}}\left[\frac{d^{n}}{d y^{n}} \log \hat{\mu}(y)\right]_{y=0}= \begin{cases}c, & n=1, \\ s_{n-2}(\lambda), & n \geqslant 2 .\end{cases}
$$

If $\lambda_{1} \sim \lambda_{2}$ are different we may use (1) (with $c=0$ ) to define two different infinitely divisible probabilities $\mu_{1}, \mu_{2} \in \mathscr{M}^{*}(\mathbf{R})$, and from (2) it is easily seen that $\mu_{1} \sim \mu_{2}$.

Conversely, if $\mu_{1} \sim \mu_{2}$ are different infinitely divisible measures, then the corresponding measures $\lambda_{1}, \lambda_{2}$ given by (1) are different and satisfy $\lambda_{1} \sim \lambda_{2}$ by (2). This shows that if $\mu$ is determinate then so is $\lambda$. We give an example of an indeterminate infinitely divisible $\mu$, for which $\lambda$ is determinate, and get, as asserted, that $\mu$ is the only infinitely divisible measure in $[\mu]$.

Let $\sigma$ be the measure constructed in Lemma 2.2 and define

$$
\mu=e^{-1}\left(\varepsilon_{0}+\sigma+\frac{1}{2} \sigma * \sigma+\cdots+\frac{1}{n !} \sigma^{* n}+\cdots\right) .
$$

Then $\mu$ is infinitely divisible and majorizes a multiple of $\sigma^{* 3}$, hence indeterminate. Since $\log \hat{\mu}=\hat{\sigma}-1$, we see from (1) that $\lambda=x^{2} \sigma$ (and $c=\int x d \sigma(x)$ ), so $\lambda$ is determinate.

3. Carleman's condition. A well-known sufficient condition for $\mu \in \mathscr{M}^{*}(\mathbf{R})$ to be determinate is the Carleman condition

$$
\sum_{n=0}^{\infty}\left(s_{2 n}(\mu)\right)^{-1 / 2 n}=\infty .
$$

As shown by the measures in 2.3(b), condition (C) is not necessary.

It is worth noticing that San Juan in 1936 (cf. [14]) showed that Stieltjes' famous indeterminate measure $\mu$ with density $\exp \left(-x^{1 / 4}\right)$ on $[0, \infty[$ can be decomposed as $\mu_{1}+\mu_{2}$ with $\mu_{1} \perp \mu_{2}$ such that (C) is satisfied for both $\mu_{1}$ and $\mu_{2}$. His construction applies to any indeterminate measure on $[0, \infty[$. 
3.1. Proposition. Let $\mu, \nu \in \mathscr{M}^{*}(\mathbf{R})$. If (C) holds for $\mu * \nu$ then it also holds for $\mu$ and $\nu$.

It is possible that (C) holds for $\mu$ and $\nu$ but that $\mu * \nu$ is indeterminate.

Proof. The first part of the result is due to Devinatz [5], and the last part follows from San Juan's construction. In fact, if $\mu=\varepsilon_{0}+\mu_{1}, \nu=\varepsilon_{0}+\mu_{2}$ then (C) holds for $\mu$ and $\nu$, but $\mu * \nu$ majorizes the Stieltjes measure and is therefore indeterminate.

3.2. Proposition. If (C) holds for $\mu \in \mathscr{M}^{*}(\mathbf{R})$ then it also holds for $\mu * \mu$.

Proof. We may assume $\mu(\mathbf{R})=1$. By Hölder's inequality we have, for $0 \leqslant k \leqslant 2 n$,

$$
\left|s_{k}(\mu)\right| \leqslant s_{2 n}(\mu)^{k / 2 n},
$$

and, hence,

$$
\begin{aligned}
s_{2 n}(\mu * \mu) & =\sum_{k=0}^{2 n}\left(\begin{array}{c}
2 n \\
k
\end{array}\right) s_{k}(\mu) s_{2 n-k}(\mu) \\
& \leqslant \sum_{k=0}^{2 n}\left(\begin{array}{c}
2 n \\
k
\end{array}\right)\left(s_{2 n}(\mu)\right)^{k / 2 n}\left(s_{2 n}(\mu)\right)^{(2 n-k) / 2 n} \\
& =\left(2\left(s_{2 n}(\mu)\right)^{1 / 2 n}\right)^{2 n},
\end{aligned}
$$

which shows the assertion.

3.3. Corollary. Let $\mu \in \mathscr{M}^{*}(\mathbf{R})$ be an infinitely divisible probability and let $\left(\mu_{t}\right)_{t>0}$ be the convolution semigroup such that $\hat{\mu}_{t}=\hat{\mu}^{t}$ for $t>0$. If (C) holds for one of the measures in $\left(\mu_{t}\right)_{t>0}$, it holds for all.

Proof. If (C) holds for $\mu_{t_{0}}$, it holds for $\mu_{t}$ for $t=2^{n} t_{0}, n=1,2, \ldots$, and hence for all $t$ by Proposition 3.1.

3.4. Remark. Let $\left(\mu_{t}\right)_{t>0}$ be a convolution semigroup as above. It follows by Theorem 1.3 that if $\mu_{t_{0}}$ is determinate then so is $\mu_{t}$ for $t \leqslant t_{0}$. If $\lambda$ is the measure corresponding to $\mu_{1}$ in (1) then $t \lambda$ corresponds to $\mu_{t}$. If $\lambda$ is indeterminate then all measures in $\left(\mu_{t}\right)_{t>0}$ are indeterminate. We have not been able to decide if it is possible that $\mu_{t}$ is determinate for $t<t_{0}<\infty$ and $\mu_{t}$ is indeterminate for $t>t_{0}$. If such an example exists then $\lambda$ must be determinate.

ACKNOWLEDGEMENT. The author wishes to thank the referee for several useful comments on an earlier version of this paper. After the completion of the first version we received the manuscript [3] which contains an example of two determinate measures $\mu$ and $\nu$ such that $\mu * \nu$ is $m$-canonical for given $m$.

\section{REFERENCES}

1. N. I. Akhiezer, The classical moment problem, Oliver and Boyd, Edinburgh, 1965.

2. C. Berg and J. P. R. Christensen, Density questions in the classical theory of moments, Ann. Inst. Fourier (Grenoble) 31 (1981), 99-114.

3. H. Buchwalter and G. Cassier, Mesures canoniques dans le problème classique des moments, Ann. Inst. Fourier (Grenoble) 34 (1984), 45-52.

4. La paramétrisation de Nevanlinna dans le problème des moments de Hamburger, Expo. Math. 2 (1984), 155-178. 
5. A. Devinatz, On a theorem of Lévy-Raikov, Ann. Math. Statist. 30 (1959), 583-586.

6. P. Diaconis and D. Ylvisaker, Quantifying prior opinion, Proc. Second Valencia Conf. on Bayesian Statistics (H. Bernardo, et al., eds.), 1984.

7. R. G. Douglas, On extremal measures and subspace density, Michigan Math. J. 11 (1964), 243-246.

8. V. P. Havin et al. (editors), Linear and complex analysis problem book. 199 Research problems. Lecture Notes in Math., vol. 1043, Springer-Verlag, Berlin and New York, 1984.

9. C. C. Heyde, Some remarks on the moment problem. II, Quart. J. Math. Oxford Ser. (2) 14 (1963), $97-105$.

10. H. J. Landau, The classical moment problem: Hilbertian methods, J. Funct. Anal. 38 (1980), 255-272.

11. E. Lukacs, Characteristic functions, Griffin, London, 1960.

12. L. C. Petersen, On the relation between the multidimensional moment problem and the one-dimensional moment problem, Math. Scand. 51 (1982), 361-366.

13. M. Riesz, Sur le problème des moments, Troisième Note, Ark. Mat. Astronom. Fys. 17 (1923), No. 16.

14. R. San Juan, Sur le problème de Watson dans la théorie des séries asymptotiques et solution d'un problème de Carleman de la theorie des fonctions quasianalytiques, Acta Math. 75 (1943), 247-254.

15. J. A. Shohat and J. D. Tamarkin, The problem of moments, Math. Surveys, vol. 1, Amer. Math. Soc., Providence, R. I., 1943.

Matęmatisk Institut, Universitetsparken 5, 2100, Copenhagen $\varnothing$, Denmark 